\title{
Accounting for the Effects of Verticals in Geodesic Surveys in the Mountains
}

\author{
Gagayeva Kh.R. \\ Faculty of construction \\ Grozny State Oil Technical University \\ named M.D. Millionshikova \\ Grozny, Russia \\ gaga_diamond@mail.ru
}

\begin{abstract}
The possibility of using an auxiliary ellipsoid to reduce the results of geodetic measurements with the aim of transferring coordinates to the surface of the reference ellipsoid is considered. An analysis of the accuracy showed that such a technique would reduce the effect of steep line dodging.
\end{abstract}

Keywords-vertical, normal vector to ellipsoid surface, reduction, deviation of verticals, radius of curvature

\section{INTRODUCTION}

Since, in the Gauss projection, the coordinates of the points are rigidly related to the dimensions of a reference spheroid at a geodetic height equal to zero, and the construction site is generally much higher, a very important source of geodetic measurements distortions is associated with the geodetic height. It is the effect of steep deviation lines, since the results of geodetic measurements are reduced to an ellipsoid along the normal to the ellipsoid, and geodesic tools in the process of field measurements are established along a vertical.

On average, in the mountainous conditions of the Caucasus, the deviation of verticals can exceed 20", which, with a geodetic height of even $500 \mathrm{~m}$, shifts the projection of a point on the reference spheroid surface by $50 \mathrm{~mm}$. And since in the mountains, the deviation of verticals can fluctuate significantly, so the magnitude of the distortion does not remain constant. This fact is only stated in the scientific and technical literature, but there are no practical recommendations for carrying out engineering and geodetic works to reduce the effect of vertical deviations. This circumstance significantly reduces the effectiveness of the use of high-precision measuring instruments in mountainous conditions.

The situation is complicated by the fact that the coordinates of the points of the state geodetic network in the Gauss coordinate system belong to the reference spheroid surface, the satellite measurement results are attributed to the PZ-90 or WGS-84 Cartesian coordinate system, and the real object is constructed at a geodetic height significantly different from the reference spheroid especially in mountainous areas.

An equally important source of distortion is the removal of the construction site in height from the reference spheroid.

\author{
Gayrabekov I.G. \\ Faculty of construction \\ Grozny State Oil Technical University \\ named M.D. Millionshikova, \\ Kh. Ibragimov Complex Institute of the Russian Academy \\ Sciences, Grozny, Russia \\ ibragim.ggni@mail.ru
}

The possibility of influence reducing of the abovementioned sources of distortion due to the influence of verticals evasion and the height of the construction site from the reference spheroid is studied.

\section{METHODS AND MATERIALS}

To study the possibility of reducing the influence of sources of distortion due to the influence of vertical evasion and the removal of the construction site in height from the reference spheroid, the using the mathematical apparatus of differential and integral calculus of functions are proposed.

The magnitude of the above distortion is characterized by the formula

$$
\Delta S=S \frac{H}{R},
$$

where $H$ - the geodesic height of the object.

The magnitude of such distortions for a segment $1 \mathrm{~km}$ long is shown in Figure 1. As can be seen from the graph, the distortions caused by the removal of the construction site from the reference spheroid become unacceptable even at a geodetic height of $200 \mathrm{~m}$ and catastrophic for large geodesic heights, and this distortion is impossible, as shown in Figure 1.

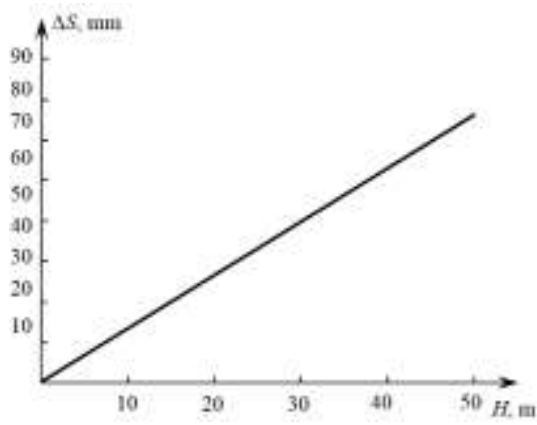

Fig. 1. Distortion dependence of the line length which is $1 \mathrm{~km}$ from geodetic height.

Since surveyors, as a rule, know only the normal height, the geodesic height can be calculated by the formula:

$$
H=H^{\gamma}+\zeta,
$$

where $H^{\gamma}$ - normal height; $\zeta$ - height anomaly. 
In Russia, the elevation anomaly varies widely from $-8 \mathrm{~m}$ in the Caspian lowland to $+50 \mathrm{~m}$ in Vladivostok. This means that in Vladivostok, even on the coast of the ocean, where the normal height is close to zero, the distortion can reach $7 \mathrm{~mm}$ per kilometer of line length.

In order to reduce distortion, it is useful to know the value of the height anomaly in the area of engineering and geodetic works. Since real objects, as a rule, are built at a significant geodetic height, a special method is needed for processing the results of geodetic measurements, which would allow combining the results of calculations of geometric parameters along the coordinates on the surface of a reference spheroid with field measurements of the same parameters with geodetic instruments. The coordinates of the points are fixed with geodesic marks on the ground surface, and the coordinates of these points refer to the surface of the reference spheroid. As a result, the real horizontal distance, which registers the distance meter $\mathrm{AB}$, is not equal to the distance $\mathrm{A}^{\prime} \mathrm{B}^{\prime}$, calculated from the coordinates (Figure 2).

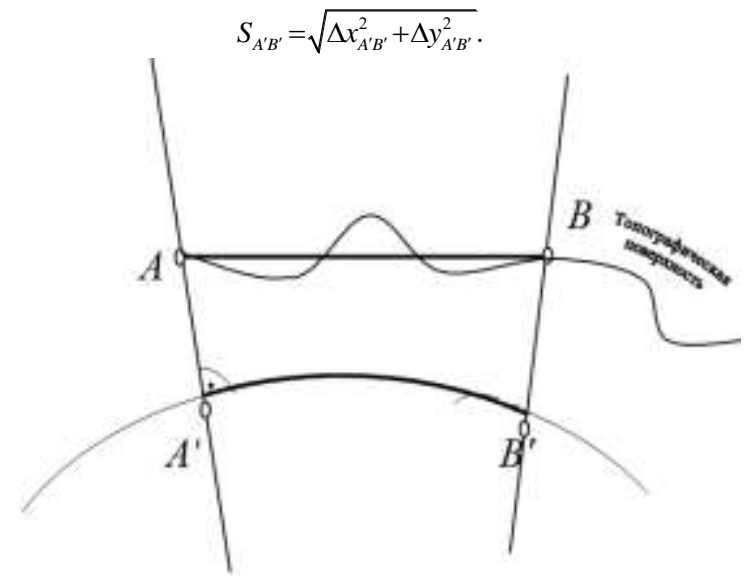

Fig. 2. Real horizontal distance of $\mathrm{AB}$ is not equal to the distance $\mathrm{A}^{\prime} \mathrm{B}^{\prime}$.

Currently, the following practice of performing engineering and geodetic works in the construction of various objects is widespread. At first, according to the design coordinates, the main axes of the object are carried out in nature. At the same time around the object should be located geodetic points, creating the so-called center network. The coordinates of the center network points are determined in the local coordinate system. In this case, it can combine only one point of the local network with the point of the State geodetic network and the direction angle of only one side. This technique is used to avoid distortion of field measurements as a result of network equalization, since field measurements are performed at a real geodetic height, and the coordinates of points of the State geodetic network are defined on the surface of the reference spheroid, where the geodetic height is zero. This can be illustrated in Figure 3 [8].

Without considering the actual geodetic height in the construction of extended objects, mutual economic claims may arise, since the actual length of the extended object will always be greater than the design length, which is calculated by coordinates. So, for example, the actual length of a 1000 $\mathrm{km}$ long road, laid at an average geodetic height of $300 \mathrm{~m}$, will be almost $50 \mathrm{~m}$ more than the design one. In mountain conditions, these distortions will be even more noticeable. At an average altitude of $1000 \mathrm{~m}$, the actual road length of only $100 \mathrm{~km}$ will be almost $16 \mathrm{~m}$ longer than the design value. With expensive construction work in the mountains, the cost of such an "additional" site will be substantial. In this case, difficulties may arise during the construction of high-rise objects [1].

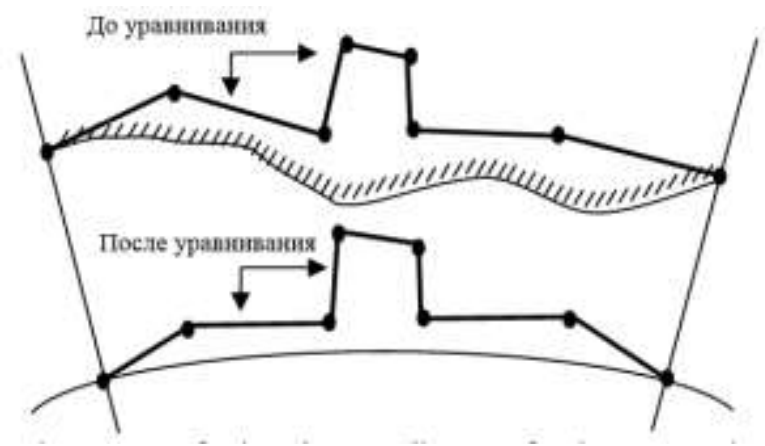

Fig. 3. Transferring the coordinates of polygonometric points to the reference surface as a result of equalization.

In modern practice of performing engineering and geodetic works, both electronic total stations and satellite receivers are equally used. However, considering the distortions inherent in the Gaussian conformal projection must have various methodological techniques. The standard errors of the difference in coordinates determined from the results of phase measurements are much smaller, and sometimes an order of magnitude smaller than the standard errors of the difference of coordinates of the same points in the state geodetic coordinate systems [10]. As a result, there is a problem when converting coordinates; the results of more accurate measurements are forced to rely on a less accurate network. At the same time, changing the coordinates of the points of the State Geodesic Network is not strongly recommended, since a huge amount of topographic surveys, layout works, executive surveys, cadastral documents was made from the points of the State Geodetic Network. With satellite measurement methods, the high accuracy of determining the differences in the coordinates of points, declared by the manufacturer of satellite receivers, is observed only in the Cartesian coordinate system PZ-90 or WGS-84. Engineering and geodetic networks are created in other, significantly different coordinate systems. In the transition from Cartesian coordinates to the coordinates of engineering and geodetic networks, the transformed coordinates can be adversely affected by inaccurate knowledge of the points height anomaly, the errors in determining the coordinates of points from the results of code measurements, the error of points coordinates of engineering geodesic networks [9].

As noted earlier, with the concentration of points in a planned geodetic network, for example, by the method of polygonometry, the reduction of measured values to the reference spheroid is not performed even at significant geodetic heights. But, if such geodetic constructions are based on points whose coordinates are calculated on the ellipsoid 
surface, then as a result of equalizing such constructions, the coordinates of the thickening network points will also belong to the surface, as shown in Figure 3. However, the rigor of equalizing the measurement results will be violated, since the residuals will contain not only field measurement errors. For example, with an average geodetic height $H=200 \mathrm{~m}$, with a stroke of only $S=1 \mathrm{~km}$, the error $\delta S$ will exceed $30 \mathrm{~mm}$.

This means that modern high-precision measuring instruments should be accompanied by qualitative methods for processing measurement results, especially since this error can increase repeatedly under mountain conditions [2-6].

Even with relatively small values of the geodetic heights of the construction site, distortions of the geodetic measurements results caused by the curvature of the Earth can exceed the accuracy of measurements with modern geodetic instruments [7]. Consequently, scientifically-based guidelines are needed to reduce arising distortions to acceptable values. Recommendations to use an auxiliary surface of relevance without a clearly defined concept of what the surface of relevance is, and, therefore, without strict mathematical support of this concept cannot be considered a universal and acceptable technique.

In the work [2], it is recommended to use an ellipsoid with modified values of the major and minor axes as a surface according to the formula:

$$
\begin{aligned}
& a^{\prime}=a \mu ; \\
& b^{\prime}=b \mu,
\end{aligned}
$$

where $\mathrm{a}$ and $\mathrm{b}$ - major and minor semiaxes of relevance ellipsoid (in Russia for engineering and geodetic works it is Krasovsky's ellipsoid);

$$
\mu=1+\frac{H_{a v e}}{R}-\text { scale factor; }
$$

$H_{\text {ave }}$ - average geodesic height at the construction site; $R-$ average radius of curvature of the Earth in the area of geodetic works.

This technique allows to ensure that the surface of the auxiliary ellipsoid passes at the height of the construction site; the geodesic height is sharply reduced, but all the mathematical relationships between the ellipsoidal coordinate system and coordinates in the Gauss projection are preserved.

Such a technique can reduce not only distortions introduced by a significant amount of geodesic height, but also errors caused by the deviation of verticals. But for this it is necessary to prove that the errors of the geodesic height will not lead to noticeable distortions. The fact is that in the transition from ellipsoidal coordinates to Cartesian, when calculating a geodetic height, knowledge of the height anomaly is required. And the transition from Cartesian coordinates to ellipsoidal one is performed without the participation of a geodetic height.

Consider that as the source material we have the coordinate points of the state geodetic network in the coordinate system, for example, SK-95 or GSK 2011. On flat rectangular coordinates the ellipsoidal coordinates are calculated. In these formulas, the values of geodetic heights of points are not used, therefore, the calculated latitudes and longitudes will not be distorted by errors of geodetic heights.
The next stage is the calculation of the Cartesian coordinates of the points $-X, Y, Z$.

The transition from ellipsoidal coordinates to spatial rectangular coordinates is carried out according to the formulas:

$$
\begin{gathered}
X=(N+H) \cos B \cos L ; \\
Y=(N+H) \cos B \sin L ; \\
Z=(N+H) \sin B-e^{2} N \sin B,
\end{gathered}
$$

In the practice of engineering and geodetic works, surveyors only know the normal height $H^{\gamma}$. The transition from normal to geodesic height is possible using the formula

$$
H=H^{\gamma}+\zeta
$$

where $\zeta_{-}$- height anomaly.

Usually, the height anomaly of surveyors is unknown or very close to the known. The magnitude of the height anomaly is significant and varies in Russia within wide limits: from $8 \mathrm{~m}$ in the Caspian Depression to $+50 \mathrm{~m}$ in Vladivostok.

Perform an analysis of the accuracy of the transition to the auxiliary surface of relevance and back from the error introduced by the inaccurate value of the height anomaly. To determine the dependence of the errors in the calculation of Cartesian coordinates on the errors of the height anomaly it is necessary to differentiate formulas (6-8) regarding [9]:

$$
\begin{gathered}
d X=d \zeta \cos B \cos L ; \\
d Y=d \zeta \cos B \sin L ; \\
d Z=d \zeta \sin B .
\end{gathered}
$$

After the Cartesian coordinates of a point are calculated, its curvilinear coordinates $\mathrm{B}^{\prime}$ and $\mathrm{L}^{\prime}$ are defined on the auxiliary ellipsoid with parameters $a^{\prime}$ and $b^{\prime}$, see $[4,5]$. The longitude of points on the auxiliary ellipsoid is calculated by the formula

$$
\operatorname{tg} L=\frac{Y}{X} .
$$

Let find out the dependence of longitude errors $L$ on coordinate errors (10)-(12), due to errors in the value of the height anomaly. For this purpose, we differentiate (13) and obtain

$$
\frac{d L}{\cos ^{2} L}=\frac{d Y}{X}-\frac{Y d X}{X^{2}} .
$$

Considering (10) and (11) the expression (14) is written in the following form

$$
d L=\frac{\cos B \cos ^{2} L \sin L}{X} d \zeta-\frac{Y \cos B \cos ^{3} L}{X^{2}} d \zeta .
$$

Using the formula

$$
Y=X \operatorname{tg} L,
$$

received from (13) and covert (15) into

$$
d L=\frac{\cos B \cos ^{2} L \sin L}{X} d \zeta-\frac{\operatorname{tg} L \cos B \cos ^{3} L}{X} d \zeta .
$$

Any observer can easily ascertain that the result is (16)

$$
d L=0 .
$$

Indeed, the point longitude is not related to the geodesic height and, as shown by the accuracy analysis performed, even though the Cartesian coordinates of point $X, Y, Z$ are computed with errors (10)-(12), the point longitude on the secondary reference surface does not contain distortions (17).

Perform an estimate of the accuracy of the calculated latitude on the secondary reference surface depending on the 
error in the height anomaly. The formula for calculating latitude $B$ is

$$
\operatorname{tg} B=\frac{Z}{\sqrt{X^{2}+Y^{2}}}\left(1+\frac{e^{2} N}{Z} \sin B\right) .
$$

According to the equitation (5) we have

$$
\frac{\sin B}{Z}=\frac{1}{\left(1-e^{2}\right) N+H} \text {. }
$$

Substituting expression (19) into formula (18), we get

$$
\operatorname{tg} B=\frac{Z}{\sqrt{X^{2}+Y^{2}}}\left(1+\frac{e^{2} N}{\left(1-e^{2}\right) N+H}\right),
$$

The formula [20] we write in the form

$$
\operatorname{tg} B=\frac{Z}{\sqrt{X^{2}+Y^{2}}}\left\{1+\frac{e^{\prime 2}}{1+\frac{H}{\left(1-e^{2}\right) N}}\right\} .
$$

Since the term of the formula (21) $\frac{H}{\left(1-e^{2}\right) N}$ is small and $1>>\frac{H}{\left(1-e^{2}\right) N}$, we use the decomposition in a series, limited to members of the first order of smallness

$$
\operatorname{tg} B=\frac{Z}{\sqrt{X^{2}+Y^{2}}}\left\{1+e^{\prime 2}-\frac{e^{\prime 2} H}{\left(1-e^{2}\right) N}\right\} .
$$

Considering

$$
e^{\prime 2}>>\frac{e^{\prime 2} H}{\left(1-e^{2}\right) N}
$$

but $e^{\prime 2} \approx 0.0067$ to assess the accuracy, it is quite possible to simplify formula (19)

$$
\operatorname{tg} B \approx \frac{Z}{\sqrt{X^{2}+Y^{2}}} .
$$

In order to assess the accuracy, we differentiate (23), remembering that Cartesian coordinates contain inaccurate information about the height anomaly:

$$
\frac{d B}{\cos ^{2} B}=\left(X^{2}+Y^{2}\right)^{3 / 2}\left[\left(X^{2}+Y^{2}\right) d Z-Z X d X-Z Y d Y\right] .
$$

Summing the squares of formulas (6) and (7), we get

$$
X^{2}+Y^{2}=(N+H)^{2} \cos ^{2} B,
$$

and considering (12) a member of formula (24), standing in square brackets, takes the form:

$$
\left(X^{2}+Y^{2}\right) d Z=(N+H)^{2} \cos ^{2} B \sin B d \zeta .
$$

In addition,

$$
X d X+Y d Y=(N+H)\left(\cos ^{2} B \cos ^{2} L+\cos ^{2} B \sin ^{2} L\right) d \zeta=(N+H) \cos ^{2} B d \zeta,
$$

and considering (8) we get:

$$
Z(X d X+Y d Y)=(N+H)^{2} \cos ^{2} B \sin B d \zeta-e^{2} N \cos ^{2} B \sin B .
$$

Therefore, the terms in square brackets of formula (24) can be represented as:

$$
\left(X^{2}+Y^{2}\right) d Z-Z(X d X+Y d Y)=e^{2} N \cos ^{2} B \sin B .
$$

Substituting (27) in (25), we get:

$$
d B=\frac{e^{2} N(N+H) \cos ^{4} B \sin B}{\left(X^{2}+Y^{2}\right)^{3 / 2}} d \zeta .
$$

Since from (25) it follows that

$$
\left(X^{2}+Y^{2}\right)^{3 / 2}=(N+H)^{3} \cos ^{3} B,
$$

we bring (28) to the form

$$
d B=\frac{e^{2} N \sin 2 B}{2(N+H)^{2}} d \zeta
$$

To assess the accuracy of the formula (29) can be easily simplified, since $\frac{N}{(N+H)^{2}} \gg \frac{1}{a}$, and given that $\frac{e^{2}}{2} \approx 0.0033$,

and expressing the magnitude $d B$ in the angular measure, we get

$$
d B=0.0033 \frac{\sin 2 B}{a} d \zeta .
$$

For latitude $B=45^{\circ}$, expressing the differential of latitude in angular seconds

$$
d B^{\prime \prime}=10^{-4} d \zeta,
$$

where $d \zeta$ - an error of anomaly height in meters.

In the transition from ellipsoidal coordinates to coordinates in the Gauss projection, the main term, the calculation of which may cause the most significant distortions, is

$$
x \approx a^{\prime} B .
$$

To assess the accuracy we differentiate (32), we have

$$
d x=a d B,
$$

and considering (30) we get

$$
d x=0.0033 \sin 2 B d \zeta \text {. (34) }
$$

As can be seen from the formula (34), the distortion of coordinates when moving to the surface of relevance is insignificant, and this technique should be used when processing the results of geodetic measurements in mountain conditions.

\section{CONCLUSION}

Thus, it can be argued that when processing the results of geodetic measurements in mountain conditions, the use of an auxiliary surface in the form of an ellipsoid with parameters at which the ellipsoid surface coincides with the surface of the construction site can significantly reduce the distortions due to the deviations of verticals and at the same time eliminate with large values of geodetic heights. This is explained by the fact that the geodesic height decreases sharply, and the linear displacement of coordinates caused by the evasion of verticals becomes neglected, and the transition from the secondary reference surface to the reference spheroid surface does not occur along the vertical, but along the normal.

\section{References}

[1] I.G. Gayrabekov, Yu.I. Pimshin, A.R. Gubeladze, "Method for determining deformation surfaces", Reports of the North Caucasus Universities. Technical Sciences, pp. 125-127, 2008.

[2] I.G. Gayrabekov, A.R. Gubeladze, I.Yu. Pimshin, P.F. Litvinova, "Calibration method of electronic rangefinders and total stations," Reports of the North Caucasus Universities. Technical Sciences, Special Issue. pp. 113-116, 2008.

[3] I.G. Gayrabekov, "Theoretical calculations Algorithm of technogenic subsidence of the earth surface of the developed fields of the TerekCaspian trough," Materials of the All-Russian scientific-practical conference "Science, education and production", dedicated to the 95th 
anniversary of Acad. M.D. Millionshchikov. Grozny. pp. 216-224, 2008.

[4] I.G. Gayrabekov, I.Yu. Pimshin, A.I. Gayrabekova, M-B.I. Gayrabekov, Kh.R. Gagayeva, "Study of the building geometry," Youth, science, innovation. Materials of the V All-Russian Scientific and Practical Conference. pp. 164-170, 2016.

[5] I.G. Gayrabekov, Yu.I. Pimshin, "Industrial research of methods for diagnosing the technical condition of buildings and structures based on geodetic measurements," Proceedings of the Grozny State Oil Technical University. Acad. M.D. Millionshchikova. 5. pp. 105-116, 2016.

[6] I.G. Gayrabekov, Yu.I. Pimshin, "Determination of the stress-strain state of buildings and structures by geodetic methods," Materials of the scientific-practical conference Construction, Rostov-na-Donu: RGSU. pp. 100-105, 2006.

[7] E.B. Klyushin, I.M. Kravchuk, Tkhan' Chyong Chin', Chan Kuang Khok, "Accounting for the effects of the earth curvature at geodetic works," Reports of universities. Geodesy and aerial photography, 1. pp. 11-14, 2012.

[8] E.B. Klyushin, I.G. Gayrabekov, E.Yu. Markelova, V.V. Shlapak, Satellite measurement methods in geodesy. Part 3. Tutorial. Moscow: Izd. MIIGAiK, 2016.

[9] E.B. Klyushin, V.V. Shlapak, E.P. Vlasenko, Khamid Frmarz Pur, "On some features of processing measurement results when solving modern geodetic tasks," Materials of the international scientific-technical conference dedicated to the 225th anniversary of MIIGAiK. Moscow, 2004

[10] Chan Kuang Khok, Chin' Tkhan' Chyong, Din' Tkhi Le Kha, "Estimation of the calculating accuracy of the coordinates increments in the Gauss-Kruger projection based on the results of satellite measurements," Geodesy and aerial photography, 2. pp. 30-35, 2017. 